\title{
An in vitro culture model to study the dynamics of colonic microbiota in Syrian golden hamsters and their susceptibility to infection with Clostridium difficile
}

Matthew Miezeiewski ${ }^{1}$, Todd Schnaufer ${ }^{2}$, Michele Muravsky², Su Wang $^{2}$, Ivette Caro-Aguilar ${ }^{2}$, Susan Secore ${ }^{2}$, David S Thiriot ${ }^{2}$, Charlie $\mathrm{Hsu}^{2,5}$, Irene Rogers ${ }^{2}$, Todd DeSantis ${ }^{3}$, Justin Kuczynski ${ }^{3}$, Alexander J Probst ${ }^{3}$, Christel Chehoud ${ }^{3}$, Rachel Steger ${ }^{4}$, Janet Warrington ${ }^{4}$, Jean-Luc Bodmer ${ }^{2,6}$ and Jon H Heinrichs ${ }^{2,7}$

${ }^{1}$ Eurofins Lancaster Laboratories Inc, Lancaster, PA, USA; ${ }^{2}$ Vaccine Research, Merck Research Laboratories, West Point, PA, USA; ${ }^{3}$ Departments of Bioinformatics, Second Genome Inc, South San Francisco, CA, USA and ${ }^{4}$ Basic Research, Second Genome Inc, South San Francisco, CA, USA

\begin{abstract}
Clostridium difficile infections (CDI) are caused by colonization and growth of toxigenic strains of C. difficile in individuals whose intestinal microbiota has been perturbed, in most cases following antimicrobial therapy. Determination of the protective commensal gut community members could inform the development of treatments for CDI. Here, we utilized the lethal enterocolitis model in Syrian golden hamsters to analyze the microbiota disruption and recovery along a 20-day period following a single dose of clindamycin on day 0 , inducing in vivo susceptibility to $C$. difficile infection. To determine susceptibility in vitro, spores of strain VPI 10463 were cultured with and without soluble hamster fecal filtrates and growth was quantified by quantitative PCR and toxin immunoassay. Fecal microbial population changes over time were tracked by 16S ribosomal RNA gene analysis via V4 sequencing and the PhyloChip assay. C. difficile culture growth and toxin production were inhibited by the presence of fecal extracts from untreated hamsters but not extracts collected 5 days post-administration of clindamycin. In vitro inhibition was re-established by day 15 , which correlated with resistance of animals to lethal challenge. A substantial fecal microbiota shift in hamsters treated with antibiotics was observed, marked by significant changes across multiple phyla including Bacteroidetes and Proteobacteria. An incomplete return towards the baseline microbiome occurred by day 15 correlating with the inhibition of $C$. difficile growth in vitro and in vivo. These data suggest that soluble factors produced by the gut microbiota may be responsible for the suppression of $\boldsymbol{C}$. difficile growth and toxin production.

The ISME Journal (2015) 9, 321-332; doi:10.1038/ismej.2014.127; published online 18 July 2014
\end{abstract}

\section{Introduction}

Clostridium difficile is a gram-positive spore-forming anaerobic bacterium commonly responsible for antibiotic-associated diarrhea in patients, mostly in hospitals or long-term care facilities. $C$. difficile infections (CDI) generally are the result of a disruption of the normal gastrointestinal microbiota by antimicrobial therapies, followed by exposure to

Correspondence: JH Heinrichs, Vaccine Basic Research, Merck Research Laboratories, West Point, PA 18902, USA.

E-mail: jon.heinrichs@sanofipasteur.com

${ }^{5}$ Current address: University of Washington, Seattle, WA 98195, USA.

${ }^{6}$ Current address: Genocea Biosciences Inc., Cambridge, MA 02140, USA.

${ }^{7}$ Current address: Sanofi-Pasteur, Swiftwater, PA 18370, USA. Received 10 October 2013; revised 23 April 2014; accepted 13 June 2014; published online 18 July 2014 spores of the bacterium, colonization and growth. Toxigenic strains of $C$. difficile produce two toxins which are responsible for a spectrum of clinical disease ranging from diarrhea to severe pseudomembranous colitis, toxic megacolon and ultimately death. C. difficile affects about 500000 Americans each year, accounting for 15 000-20000 deaths (Rupnik et al., 2009) with similar incidence reported in both Canada and Europe (Poutanen \& Simor, 2004).

Current mainstream therapies for CDI are mostly empiric and range from discontinuation of the offending antibiotic to controlled courses of narrow-spectrum antibiotics (metronidazole, vancomycin-individually or in various combinations). As these therapies have no effect on the latent spores in the gastrointestinal and the surrounding environment, nor improve the health of the colonic microbiota (if not lead to further dysregulation), the 
rate of disease recurrence is high. A clear indication that the gastrointestinal microbiota is central to the control of CDI is supported by the results of a recent double-blind randomized clinical trial where the rate of cure of recurrent CDI was increased from $31-81 \%$ in subjects given fecal transplant compared with those on vancomycin therapy (van Nood et al., 2013). A better understanding of the protective role of the commensal gastrointestinal microbiota could therefore provide critical information about how current and future antimicrobial treatments affect CDI risks as well as lead to the development of potential new therapies. Identification of the protective components that are contained in these fecal emulsions could also lead to the development of biomarkers associated with disease risk.

Previous efforts to understand the protection afforded by the commensal microbiota have demonstrated that fecal emulsions from healthy human adults retain their ability to inhibit growth and toxin production of $C$. difficile in vitro (Borriello and Barclay, 1986). To examine both the in vitro and in vivo interactions between the commensal microbiota and $C$. difficile, we used the lethal enterocolitis model in Syrian golden hamsters (Mesocricetus auratus). This model is a useful experimental tool that has been widely used to evaluate the protective potential of various prophylactic and therapeutic interventions against CDI, as well as to compare the virulence of various $C$. difficile strains (Sambol et al., 2001).

To establish whether a correlation between the composition of the commensal microbiota and susceptibility to infection exist in this model, we generated soluble extracts from feces collected from hamsters both before and after antibiotic administration and evaluated their effect on $C$. difficile growth in vitro. In parallel we challenged vaccinated hamsters with a lethal dose of a prototypic $C$. difficile strain (VPI 10463) and compared the course of disease in these animals with the growth kinetics of $C$. difficile in vitro. Finally, we examined the bacterial composition of the gut microbiota at various times post clindamycin treatment to determine the impact of antibiotic treatment on normal microbiota and shed light on the role of these gut residents on susceptibility to CDI.

\section{Materials and methods}

C. difficile strains and spore preparation

Spores were prepared from strain VPI 10463, (American Type Culture Collection, Manassas, VA, ATCC \#43255) using established methods (Long Sterling and Williams, 1958; Sorg and Sonenshein, 2008). Spores were aliquoted in water and stored frozen at $-70^{\circ} \mathrm{C}$ for up to 1 year. Spores were thawed prior to challenge and diluted in phosphatebuffered saline (PBS) to a final concentration of $1000 \mathrm{CFU} \mathrm{ml}{ }^{-1}$. Each animal received $1 \mathrm{ml}$ of C. difficile spore preparation. C. difficile selective agar plates (CDSA) (Becton Dickinson, Franklin
Lakes, NJ, USA) supplemented with sodium taurocholate (Pfaltz \& Bauer Inc, Waterbury, CT, USA) added at a concentration of $0.1 \% \mathrm{w} / \mathrm{v}$ (CDSA-T) were used for agar plate culture in vitro. Brain Heart Infusion broth (Teknova Inc, Hollister, CA, USA) containing $0.1 \% \mathrm{w} / \mathrm{v}$ taurocholate was used for liquid culture.

\section{Fecal extract preparation}

Hamster feces were collected daily pre and post treatment of animals with various doses $(30,10$ or $1 \mathrm{mg} \mathrm{kg}^{-1}$ ) of clindamycin (Cleocin, Pfizer, New York, NY, USA). All fecal samples were frozen at $-70{ }^{\circ} \mathrm{C}$ before the soluble fraction was extracted for use in experiments to determine their growth inhibition properties. Sterile extracts were prepared by dissolving the feces 1:10 w/v in PBS, followed by centrifugation at $4000 \mathrm{~g}$ for $10 \mathrm{~min}$ at $4{ }^{\circ} \mathrm{C}$ to pellet the insoluble components and the supernatants were then decanted and filter sterilized using a syringe filter $(0.22 \mu \mathrm{m})$ (Thermo Fisher Scientific, Rochester, NY, USA). Fecal extracts were added at a ratio of one part fecal extract to 99 parts medium. After spore addition, cultures were incubated in an anaerobic culture chamber conditioned using an anaerobic gas generating system (Thermo Fisher Scientific, Lenexa, KS, USA) at $37^{\circ} \mathrm{C}$ for $72 \mathrm{~h}$.

\section{C. difficile toxin enzyme immunoassay}

To measure the total concentration of $C$. difficile toxin $A$ and toxin $B$, fecal extract culture supernatants were analyzed using a C. DIFFICILE TOX A/B II Kit (TechLab, Blacksburg, VA, USA). Briefly, fecal extract culture supernatants were precipitated with 60\% ammonium sulfate $\left(\mathrm{NH}_{4} \mathrm{SO}_{4}\right)$ added from a saturated solution at $4{ }^{\circ} \mathrm{C}$. The precipitated samples were then resuspended in kit diluent and analyzed according to the manufacturer's instructions. This kit has documented toxin $\mathrm{A}$ and toxin $\mathrm{B}$ detection levels of $\geqslant 0.8 \mathrm{ng} \mathrm{ml}^{-1}$ and $\geqslant 2.5 \mathrm{ng} \mathrm{ml}^{-1}$, respectively.

\section{Quantitative PCR ( $q P C R$ )}

A qPCR assay was used to determine the number of C. difficile genome copies present in either fecal emulsions from $C$. difficile-challenged hamsters or from fecal extract supplemented broth cultures. Samples were stored at $-20^{\circ} \mathrm{C}$ until analysis by qPCR. Samples were thawed on the benchtop at room temperature and diluted 1:10 v/v with PBS prior to DNA purification. Twenty-five microliters of proteinase $\mathrm{K}\left(20 \mathrm{mg} \mathrm{ml}^{-1}\right)$ (Qiagen, Valencia, CA, USA) was added to $400 \mu \mathrm{l}$ of the suspension. Samples were mixed briefly using a vortex and placed in a water bath at $74^{\circ} \mathrm{C}$ for $10 \mathrm{~min}$ for enzymatic digestion. Samples were then transferred to an automated DNA purification robot (QiaSymphony SP, Qiagen) and purified using a QiaSymphony DNA Mini Kit (Qiagen). Samples were eluted in $200 \mu \mathrm{l}$ of elution buffer and held at $4{ }^{\circ} \mathrm{C}$ until use. QPCR primers and probes for the 
detection of $C$. difficile were based on the intergenic region of 16S ribosomal RNA (rRNA) sequences and used as previously described (Penders et al., 2005).

Amplification was carried out on a FAST enabled 7900SDS (ABI, Carlsbad, CA, USA) thermocycler in a total volume of $20 \mu \mathrm{l}$ with $750 \mathrm{~nm}$ of both primers, $166 \mathrm{~nm}$ 6-carboxyfluorescein/tetramethylrhodamine probe, $2 \times$ universal FAST Taq PCR mix (ABI) and $2 \mu \mathrm{l}$ template DNA. The following cycle parameters were used: $95{ }^{\circ} \mathrm{C}$ hold for $20 \mathrm{~s}$ followed by 50 cycles of $95{ }^{\circ} \mathrm{C}$ for $30 \mathrm{~s}$ and $60{ }^{\circ} \mathrm{C}$ for $30 \mathrm{~s}$. DNA was diluted to fit within a standard range $\left(10^{1}-10^{6}\right.$ copiesper $\left.\mu \mathrm{l}\right)$ prior to amplification in Tris-EDTA buffer and values were back-calculated according to the dilution factor. Standards used were based on a plasmid containing the $C$. difficile $16 \mathrm{~S}$ rRNA intergenic region from strain 630 and run in 10-fold serial dilutions. Quantities were interpolated from the plasmid standard curve.

\section{Animal husbandry and handling}

Male Syrian golden hamsters, $M$. auratus, (90-120 gm) (Charles River Laboratories, Stonebridge, NY, USA) were housed individually in sterilized boxes with micro isolator lids. Food, water and bedding were autoclaved prior to clindamycin treatment of animals. The cages were changed daily following $C$. difficile spore challenge. All animal experiments were approved by the Institutional Animal Care and Use Committee at Merck Research Laboratories (West Point, PA, USA).

\section{Toxoid vaccine preparation}

Vaccine for immunization of animals was prepared in the following way: lyophilized $C$. difficile toxins A and B (List Biological Laboratories, Campbell, CA, USA) were separately reconstituted and then dialyzed into $20 \mathrm{~mm}$ MOPS-HCl pH 7.4, $150 \mathrm{~mm} \mathrm{NaCl}$, at concentrations of $\sim 0.6 \mathrm{mg}$ toxin per ml. Formaldehyde was added to a concentration of $0.42 \%$, and lysine was added to a concentration of $4.3 \mathrm{mg} \mathrm{ml}^{-1}$. After an 18-day incubation at $2-8{ }^{\circ} \mathrm{C}$, the toxoids were combined with amorphous aluminum hydroxyphosphate sulfate (Merck, West Point, PA, USA) and ISCOMATRIX adjuvant (CSL Biotherapies, Inc, King of Prussia, PA, USA) in $20 \mathrm{~mm}$ HEPES, $100 \mathrm{~mm}$ sodium chloride, $\mathrm{pH} 7.5$ buffer to produce a vaccine with final concentrations of $10 \mu \mathrm{g} \mathrm{ml}^{-1}$ toxoid A, $10 \mu \mathrm{g} \mathrm{ml}^{-1}$ toxoid $\mathrm{B}, 450 \mu \mathrm{g}$ aluminum per ml, $100 \mathrm{IU} \mathrm{ml}^{-1}$ ISCOMATRIX adjuvant and a residual formaldehyde concentration of $0.015 \%$. The injection volume in hamsters was $0.2 \mathrm{ml}$ per dose.

\section{Toxoid vaccine immunization}

The animals were immunized four times at $0,21,42$ and 63 days with toxoid $\mathrm{A}$ and toxoid $\mathrm{B}$ using the intra-muscular route in the quadriceps femoris muscle. Animals were bled at each time point as well as on day 77 (2 days prior to challenge with C. difficile).
Challenge

Hamsters were treated by oro-gavage with a single dose of 1,10 or $30 \mathrm{mg} \mathrm{kg}^{-1}$ clindamycin 5 days before the administration of spores. C. difficile spores were administered to the animals by oro-gavage and the animals were monitored for symptoms (wet tail and weight loss) or death twice daily for 2 weeks.

\section{Anti-toxin A/B ELISA assays}

An ELISA assay was used to measure the serum immunoglobulin $\mathrm{G}$ titer of immunized animals for toxin A and B. Thermo Reacti-Bind (Thermo Lab Systems, Waltham, MA, USA) 96-well plates were coated with $C$. difficile toxin A or toxin B (both from List Biological Laboratories) at $50 \mathrm{ng}$ per well and 25 ng per well, respectively, in PBS and incubated overnight at $4{ }^{\circ} \mathrm{C}$. Wells were washed with $300 \mu \mathrm{l}$ of PBS containing $0.05 \% \mathrm{v} / \mathrm{v}$ Tween 20 (PBS-T) three times, and unbound sites blocked with $100 \mu \mathrm{l}$ of blocking/dilution solution (PBS containing 1\% bovine serum albumin (Sigma, St Louis, MO, USA)) by incubation for $1.5 \mathrm{~h}$ at room temperature. Hamster sera were serially diluted (fivefold steps) on the plates and incubated at room temperature for $1.5 \mathrm{~h}$. Plates were washed with $300 \mu \mathrm{l}$ of PBS-T three times and incubated with goat anti-hamster immunoglobulin $G$ horse-radish peroxidase conjugated antibody (Abcam, Cambrige, MA, USA) for $1 \mathrm{~h}$ at room temperature. Following an additional wash with $300 \mu \mathrm{l}$ of PBS-T, $50 \mu \mathrm{l}$ of 3,3',5,5'-tetramethylbenzidine substrate (Thermo Labsystems, Milford, MA, USA) was added and after $15 \mathrm{~min}$, the reaction was stopped by the addition of $50 \mu \mathrm{l}$ of $0.4 \mathrm{~N}$ sulfuric acid (Fisher Scientific, Pittsburg, PA, USA) and absorbance was measured at $450 \mathrm{~nm}$.

\section{Microbial flora profiling}

Microbiota from fecal specimens were profiled using Second Genome's Microbiome Signature Discovery service (San Bruno, CA, USA), which included both PhyloChip and Illumina sequencing assays to track microbial population dynamics across the specimens as detailed in the Supplementary Methods. Briefly, for PhyloChip G3 assay profiling, the V1 through the V9 regions of the 16S rRNA genes were amplified, and $\sim 3.0 \times 10^{10}$ molecules (500 ng) from each sample were hybridized to the microarray displaying 1016064 oligonucleotide probes complementing all known bacterial and archaeal taxa as described in previous work (Hazen et al., 2010). A total of 934 empirical operational taxonomic units (OTU) were found in one or more samples and were taxonomically annotated against Greengenes (DeSantis et al., 2006; McDonald et al., 2012). A hybridization score was calculated for each sample from the mean ranked fluorescent intensities among the multiple probes assigned to each empirical OTU.

For V4 sequencing assay profiling, the V4 region of the 16S rRNA genes was amplified using fusion primers as previously described (Caporaso et al., 2011), 
and then pooled for sequencing using the MiSeq (Illumina, San Diego, CA, USA) instrument. Using the software QIIME (Kuczynski et al., 2011), UCLUST (Edgar, 2010) and MOTHUR (Schloss et al., 2009), sequences were clustered into 3913 reference OTUs and assigned taxonomic classification from the Greengenes database. To normalize the sequence counts per specimen, 18946 sequences were randomly drawn without replacement from each sample, equivalent to $7.0 \times 10^{-6} \mathrm{ng}$ of the PCR product. The count of 18946 was determined by the sample with the least number of sequences retained after clustering to reference OTUs.

All OTU abundance profiles were compared with Weighted UniFrac (Lozupone et al., 2006) and significant changes in OTU abundance patterns across hamster groups and time points were determined with the Welch or analysis of variance tests for PhyloChip or V4 sequencing, respectively. The resulting $P$-values were corrected to $q$-values with the Benjamini-Hochberg procedure.

To identify the putatively protective OTUs (PPOs) associated with C. difficile resistance, OTU abundance trajectories from both methods were filtered to those that decreased significantly after the $30 \mathrm{mg} \mathrm{kg}^{-1}$ clindamycin dose but then increased significantly by day 15 (method details in Supplementary Table S3). A significance level of $\mathrm{q}<0.05$ for PhyloChip data was chosen. Because no V4 OTUs passed all four criteria with that stringency, the $\mathrm{V} 4$ significance level of $\mathrm{P}<0.05$ was chosen. PPOs were compared with data from the Human Microbiome Project Consortium (HMP, 2012) to determine their clinical relevance.

\section{Results}

Effect of clindamycin treatment on fecal extracts in in vitro culture

We first examined the ability of fecal extracts obtained from clindamycin-treated hamsters to modulate the growth of $C$. difficile strain VPI 10463 on CDSA-T plates. Extracts were prepared from hamster feces collected before and after treatment of animals with $30 \mathrm{mg} \mathrm{kg}^{-1}$ clindamycin. As expected, in the absence of any fecal extract, spores germinated and grew on the selective agar medium. In contrast, the addition of fecal extracts from animals that had not been clindamycin treated (naïve group) yielded a mean value of 0.75 CFUs per plate (s.d. =1.5 CFUs) compared with the control which produced a mean of 97.5 CFUs per plate (s.d. = 18.82 CFUs). However, the plates containing fecal extracts made from feces collected 5 days post antibiotic treatment of animals showed no inhibition of $C$. difficile growth as demonstrated by colonies that were similar to the control in both number and size. Heat treatment or ultrafiltration (cutoff $\sim 3000 \mathrm{Da}$ ) of extracts from naïve animals completely abrogated their inhibitory effects suggesting that the compound(s) responsible for inhibition was most likely a heat-sensitive protein.

Next, a study of the kinetics of microbiota disruption by antibiotic administration was conducted during which fecal extracts were collected daily following the treatment of hamsters with clindamycin and these extracts were added to liquid cultures containing C. difficile spores. Following incubation, bacterial growth and toxin production were evaluated by quantitative $16 \mathrm{~S}$ ribosomal DNA qPCR and an enzyme immunoassay, respectively. Early time points, utilizing extracts collected from animals shortly after clindamycin treatment, were inhibitory for $C$. difficile growth, either due to residual clindamycin in the animals' feces, or due to incomplete disruption of the microbiota of animals. However, beginning at $\sim 3$ days following antibiotic treatment, extracts from treated animals were no longer capable of inhibiting the growth of C. difficile in culture and this lack of activity persisted until about day 12. These data demonstrated that administration of a single clindamycin dose to animals resulted in an 10-day long period of time during which fecal extracts were not inhibitory for C. difficile growth and subsequent toxin production. After this 10-day period the inhibitory activity of fecal extracts was restored, suggesting replenishment of the microbiota (Figure 1).

To investigate whether the magnitude of the disruption was dependent upon the dose of antibiotic, reduced doses of clindamycin were administered to hamsters ( 1 or $10 \mathrm{mg} \mathrm{kg}^{-1}$ ) and fecal extracts were prepared from these animals and compared with fecal extracts from animals treated with $30 \mathrm{mg} \mathrm{kg}^{-1}$ clindamycin. Lowering the dose of clindamycin appeared to modify the kinetics of disruption in the fecal extract assay (Figure 2) and resulted in shorter time periods during which C. difficile growth and concurrent toxin production were enabled in liquid cultures.

\section{Effect of clindamycin treatment on susceptibility of hamsters to C. difficile challenge}

Syrian hamsters are exquisitely sensitive to challenge with $C$. difficile and usually die within a few days post infection, making an in vivo evaluation of fecal microbiota difficult. However, toxoid-immunized animals have been shown to be resistant to challenge and therefore allowed us to follow animals for a full 14-day observation period to analyze changes in their microbiota utilizing various methods. Therefore, a cohort of animals were immunized with toxoids A and B (four doses, intramuscular) and were bled on day 77 and antitoxin titers to the two large clostridial toxins were determined by ELISA (Figure 3) to confirm seroconversion of the animals. As expected, the animals immunized with the toxoid vaccine had significantly higher titers to toxin A and B than the animals in the adjuvant control group. 

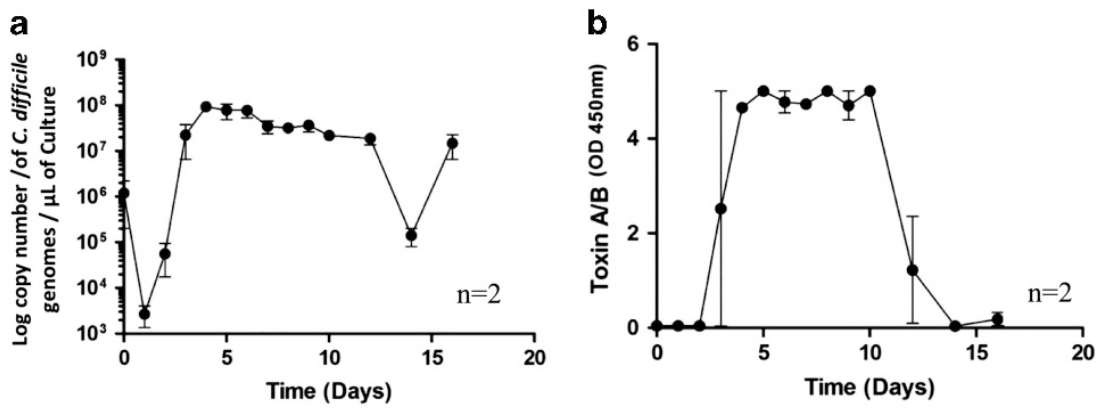

Figure 1 Culture and toxin production kinetics of $C$. difficile in vitro in the presence of fecal extracts. (a) 16S ribosomal DNA qPCR of in vitro culture containing fecal extracts from various time points post antibiotic disruption. (b) Toxin enzyme immunoassay assay measurements from the same culture as the qPCR data showing production of toxin when $C$. difficile growth occurs in vitro; error bars shows range.

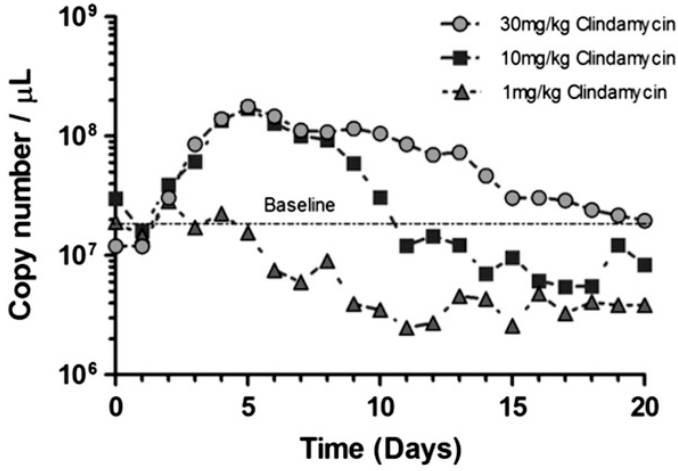

Figure 2 In vitro culture of $C$. difficile strain VPI 10463 measured by $16 \mathrm{~S}$ ribosomal DNA qPCR. The length of time associated with high $C$. difficile growth correlates with the dose of antibiotic used to generate the disruptions of the microbial flora. The in vitro culture grown in the presence of fecal extracts from the 30 (circle), 10 (square) and $1 \mathrm{mg} \mathrm{kg}^{-1}$ (triangle) of clindamycin-treated hamsters generated a 15, 10 and a 3 day permissive window in the gut microbiota, respectively. Baseline mark is geomean of the untreated hamster copy number per $\mu \mathrm{l}$ ( $N=5$ hamsters per group).

Hamsters that had been treated with either 10 or $30 \mathrm{mg} \mathrm{kg}^{-1}$ clindamycin following immunization with adjuvant alone were highly susceptible to C. difficile challenge with the majority of animals succumbing to the infection within the 14-day observation period. However, animals that were immunized with the toxoid vaccine were protected against $C$. difficile challenge following disruption with the same doses of antibiotic $(P<0.0001)$ (Figure 4a). In contrast, treatment of hamsters with a $1 \mathrm{mg} \mathrm{kg}^{-1}$ dose of clindamycin did not generate animals that were sensitive to $C$. difficile infection as the majority of hamsters in both the adjuvant and vaccine immunized groups survived challenge.

We also monitored the body weight changes following challenge of animals, which provided an indirect method of monitoring the progression of CDI in these animals. Weights of individual immunized animals were normalized to initial body weights to allow for comparison between animals (Figure 4b). A recovery point in this model was identified by locating the time point at which the hamsters began to either gain or stabilize their weight and maintain gains over several days. This time point varied depending on the dose of antibiotic given and occured on or before day 5 for the $1 \mathrm{mg} \mathrm{kg}^{-1}$ dose and post day 11 and 15 for the 10 and $30 \mathrm{mg} \mathrm{kg}^{-1}$ doses, respectively.

The impact of timing of spore introduction post antibiotic administration on animal health and survival was also investigated to assess the time period during which hamsters were susceptible to challenge. Naïve hamsters were challenged with VPI 10463 spores at 5, 9, 15 or 20 days post treatment with clindamycin ( $30 \mathrm{mg} \mathrm{kg}^{-1}$ dose). The time between antibiotic disruption and the introduction of C. difficile spores into the gut correlated with the survival of hamsters and longer intervals led to increased survival. QPCR of stool pellet emulsions also revealed that feces obtained from animals with a longer time interval between disruption and challenge had a lower overall burden of $C$. difficile bacteria (Figure 5).

To dissect the effect of antibiotic treatment on the bacterial composition of the gut, microbial profiling was conducted on fecal samples collected from hamsters either before clindamycin treatment or on days $5,9,15$ and 20 post treatment in the hopes of identifying taxa that might be associated with the inhibitory phenomenon described in Figure 1. Significant microbiome changes were observed between specimens sampled on different days post treatment $(P<0.001)$. Microbiota profiling revealed profound changes in bacterial taxa present in feces, when comparing samples obtained at day 0 and those obtained on day 5 and a subsequent return to near baseline composition by day 20 , confirming the observed in vitro and in vivo time course of susceptibility to $C$. difficile challenge. Using the microarray approach we examined bacterial taxa that demonstrated a change in density in comparison to the undisrupted state.

Distinct microbiomes were associated with the resistant, susceptible and recovered phases

From the results of the in vitro and in vivo time course experiments described above, three phases were defined: the Resistant Phase on day 0, the Susceptible Phase from days 1 to 9 and the Recovered Phase from days 10 to 20. Two 16S rRNA 

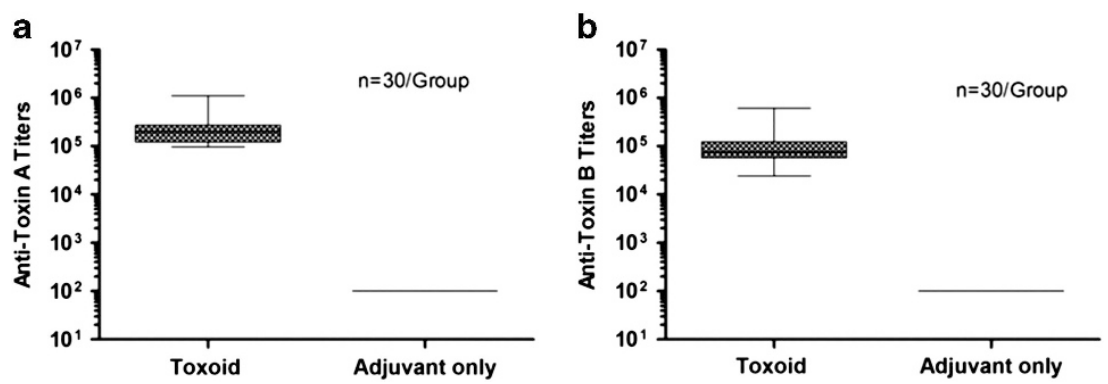

Figure 3 ELISA titers from hamsters immunized with either toxoid A/B or adjuvant only. (a) Anti-toxin A antibodies ( $P>0.0001)$ (b) Anti-toxin B antibodies $(P>0.0001)$
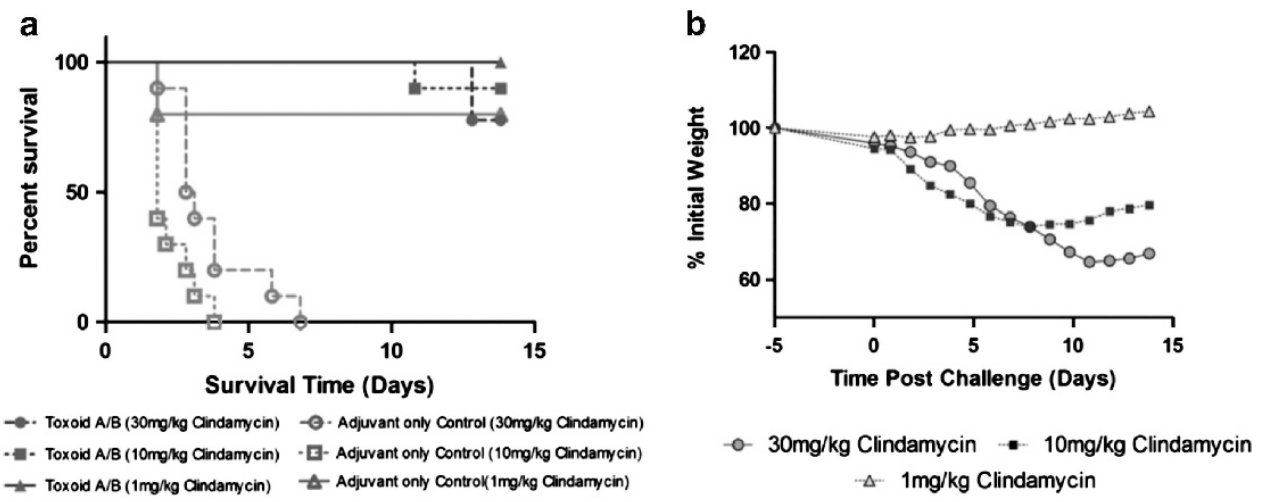

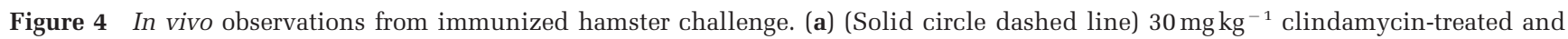
toxoid-immunized hamsters. (Solid square dashed line) $10 \mathrm{mg} \mathrm{kg}^{-1}$ clindamycin disrupted and toxoid-immunized hamsters. (Solid triangle solid line) $1 \mathrm{mg} \mathrm{kg}^{-1}$ clindamycin disrupted and toxoid-immunized hamsters. (Open circle dashed line) $30 \mathrm{mg} \mathrm{kg}{ }^{-1} \mathrm{clindamycin}$ disrupted and no vaccine immunized hamsters. (Open square dashed line) $10 \mathrm{mg} \mathrm{kg}^{-1}$ clindamycin disrupted and no vaccine immunized hamsters. (Open triangle solid line) $1 \mathrm{mg} \mathrm{kg}^{-1}$ clindamycin disrupted and no vaccine immunized hamsters (b) Percent initial weight loss averaged over the group of toxoid-immunized hamsters for each day of the challenge illustrating the recovery points in the challenge model ( $N=10$ hamsters per group).
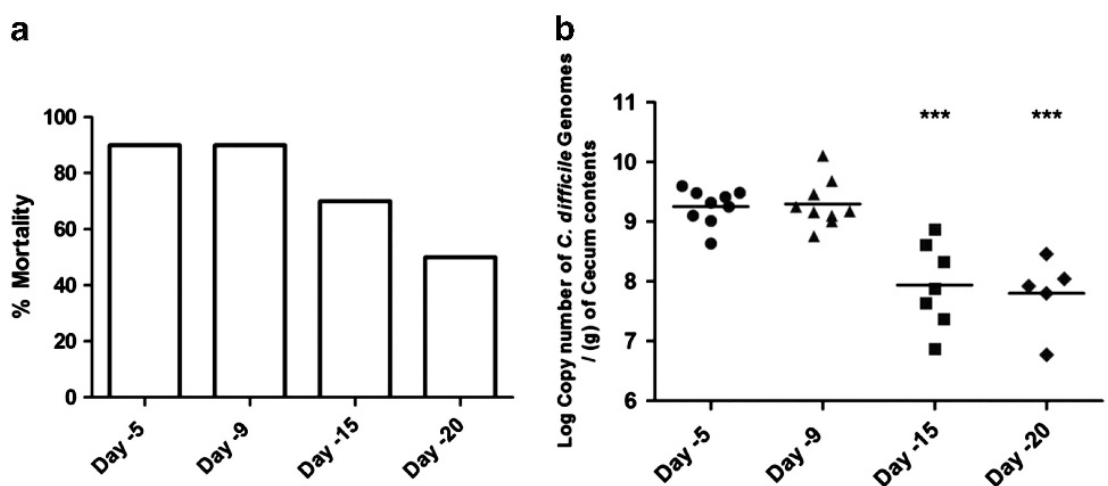

Figure 5 C. difficile introduction timing post disruption. (a) Percent mortality of hamsters by time of spore introduction. (b) 16S qPCR of cecal contents of the hamsters at time of termination. ${ }^{* *} P<0.05$ when compared with 5 days post disruption spore challenge $(N=10$ hamsters per group).

gene methods were used to track population dynamics, DNA-DNA hybridization-based PhyloChip probe and V4 Illumina MiSeq sequencing. Microbiome dissimilarity between samples was calculated with the Weighted UniFrac method (Lozupone et al., 2006) and visualized using principal coordinate analysis in Figure 6, where each point represents one sample and the distance between points represents the dissimilarity in the types and populations of microbiota within samples. The level of significance for inter-group dissimilarity was summarized in Supplementary Table S1.

A general agreement between the PhyloChip and V4 community shift data is detailed below and can be summarized with four high level observations: (1) starting gut communities were similar across all treatment groups, (2) high antibiotic dosage had a greater effect than low dosage, which had a greater 
effect than the PBS treatment, (3) recovery was slower than perturbation and was not complete by day 20 and (4) outlier identification was congruent. No significant difference was observed between the three animal treatment groups (PBS, $1 \mathrm{mg} \mathrm{kg}^{-1}$ and $30 \mathrm{mg} \mathrm{kg}^{-1}$ clindamycin) on day 0 pre treatment indicating that no group contained a starting community structure which was systematically biased. In other words, all hamsters had similar starting microbiomes. The PhyloChip results revealed that the microbiomes in the PBS-treated hamsters had significantly changed when sampled at different time points $(P=0.030)$ as seen by the drift along both principal coordinate analysis (PCoA) axes among the points colored blue, purple and light green. The V4 data did not reveal a community shift over time in the PBS cohort $(P=0.557)$. In the low dosage group, which received $1 \mathrm{mg} \mathrm{kg}^{-1}$ clindamycin, significant microbiome changes greater in magnitude than in the PBS group were demonstrated by day 5 using both technologies as evidenced by the decreases in within:between ratios in Supplementary Table S1. In the high dosage group, the community shift from day 0 to 5 was larger than those observed in the low dose group and even lower within:between ratios resulted.

Samples collected on days 5 and 9, the Susceptible Phase, in the high dosage group, were the most dissimilar from the baseline samples, which were visualized by a large change in their position along PCoA1. Both methods were in agreement in revealing that significant shifts had taken place between days 5 and 9 (PhyloChip, $P=0.038$; V4, $P=0.022$ ). Both methods also showed that sample C30_615_d09 was an outlier along the PCoA primary axis in Figure 6 compared with the other day 9 samples from the high-dose-treated hamsters. C30_615_d09 was the only sample from the Susceptible Phase observed as an outlier. Excluding C30_615_d09, the PhyloChip PCoA indicated less overlap between day 5 and 9 samples than the V4 PCoA.
The period of time from day 9 to 15 was sufficient for the high-dose hamsters to recover from their C. difficile susceptibility and the corresponding microbial community structure was observed to have shifted marginally in the V4 data $(P=0.061)$ and significantly in the PhyloChip data $(P=0.019)$ towards the baseline structure. Communities on days 15 and 20 were indistinguishable by the Adonis test due to high intra-group variance driven by hamster 616 (Figure 6). Notably, the microbiome from the high dose clindamycin treatment group remained distinct from the baseline microbiome even 20 days post treatment (PhyloChip, $P=0.013$; V4, $P=0.033$ ) and was not distinct from the day 5 microbiome of the low dose hamsters (PhyloChip, $P=0.570$; V4, $P=0.742$ ). In summary, a high dose of clindamycin resulted in a rapid microbiome perturbation and associated susceptibility to infection followed by a slower phenotypic recovery and an incomplete return to baseline microbial community structure.

\section{Antibiotic treatment affected populations within multiple phyla}

Clindamycin treatment was associated with population shifts among multiple phyla. As shown in Supplementary Figure S1 the samples from the same treatment group on the same day were observed to have reproducible proportions in overall hybridization scores and the two largest changes in phylumlevel composition were readily observed. In comparison to pre-treated and PBS-treated hamsters, hamsters treated with $30 \mathrm{mg} \mathrm{kg}^{-1}$ clindamycin proportionally lost Bacteroidetes $\left(P=2.6 \times 10^{-16}\right)$ and gained Proteobacteria $\left(P=2.8 \times 10^{-20}\right)$ by day 5 , an observation corroborated by the V4 data (Supplementary Figure S2). In fact, 18 of the 24 phyla tracked demonstrated highly significant shifts $\left(P<1.0 \times 10^{-03}\right)$ in populations (Supplementary Table S2), but considering the phylum-level data was normalized to be compositional, or additive to $100 \%$, some changes are likely not independent
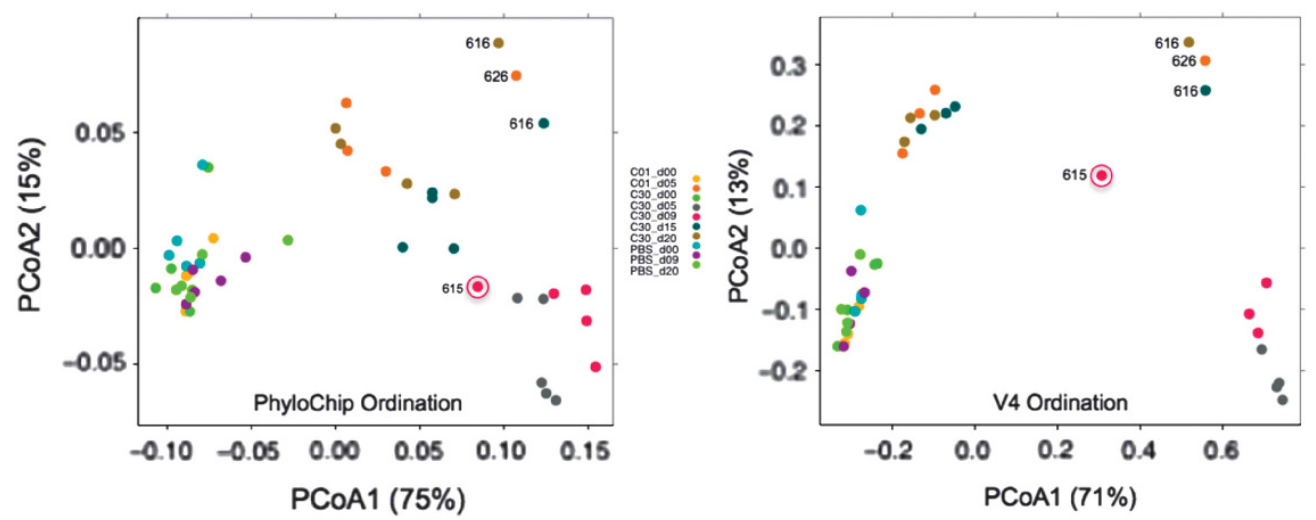

Figure 6 Sample-to-sample weighted UniFrac dissimilarity matrix ordinated by principal coordinate analysis (PCoA) along two axes. Percent of dissimilarity matrix explained by each axis is denoted in parentheses. Color legend applies to both PhyloChip (left) and V4 (right) plots. Selected samples are labeled with hamster number. Points representing sample C30_615_d09, the sole outlier in the susceptible phase, are circled in pink. 
(Friedman and Alm, 2012). Interesting phyla generally considered as environmental, such as the recently described Armatimonadetes (Tamaki et al., 2011) were not only encountered in the fecal samples but demonstrated significant increase in relative abundance after antibiotic application. In terms of fold-change, Firmicutes, Tenericutes and Actinobacteria were more stable than Bacteroidetes and Proteobacteria. The most significant change in cumulative hybridization scores was a greater threefold increase post antibiotic treatment in Fusobacteria $\left(P=2.4 \times 10^{-19}\right)$ despite this phylum ranking low in the overall proportions. In comparing the phylum composition reported by hybridization and sequencing, a general trend was determined in that the phyla observed with low proportions in the overall hybridization scores were not detected in the V4 sequencing data set.

\section{Distinct OTU population trajectories were observed between the Resistant, Susceptible and Recovered phases}

Both techniques, PhyloChip G3 and V4 sequencing, verified that multiple individual OTUs were significantly altered after the $30 \mathrm{mg} \mathrm{kg}^{-1}$ clindamycin treatment. Examining all taxa, 887 of 934 PhyloChip OTUs and 380 of $3913 \mathrm{~V} 4$ sequencing OTUs achieved a level of significance of $q<0.05$ from a three group analysis of variance test, where the three groups were defined as samples from the Resistant Phase at day 0, the Susceptible Phase at days 5 and 9 and the Recovered Phase at days 15 and 20. Outlier sample C30_615_d09 was excluded from analysis of variance tests. The population trajectories of the most significantly altered OTUs as assayed via PhyloChip and V4 sequencing revealed that OTUs followed distinct population shifts (Supplementary Figure S3). For example OTUs within Rikenellaceae and Oceanospirillales were depressed after treatment. In contrast, OTUs within Ruminococcaceae, Enterobacteriaceae, Eubacterium and others were found in a greater abundance in the amplicon community after antibiotic treatment relative to pre treatment. Regardless of the method, OTUs with significant abundance shifts were observed and the directions of population changes across phases were not the same for each OTU.

\section{Putatively protective taxa were identified}

We searched specifically for OTUs significantly reduced in population during the Susceptible Phase of days 5 and 9 when compared with the undisrupted Resistant Phase, followed by a significant increase in the Recovered Phase of days 15 and 20. An OTU meeting these two criteria was considered a PPO. In this analysis, the Resistant Phase was represented by all day 0 samples regardless of treatment arm and all PBS-treated samples regardless of time point. To remove the OTUs demonstrating the PPO pattern due to the time effect alone, those significantly decreased in abundance in PBS-treated hamsters on day 9 were excluded, as were those that significantly increased in abundance on day 20 compared with day 9. Counts of OTUs retained at each filtering step are presented in Supplementary Table S3. The search found 153 PhyloChip OTUs meeting all criteria even after the Benjamini-Hochberg correction procedure for statistical significance (Figure 7) and zero V4 sequencing OTUs evaluated with the same thresholds. Therefore, the stringency was relaxed for the V4 OTUs to utilize the $P$-values without Benjamini-Hochberg correction to yield eight OTUs (Supplementary Figure S4). The same results were obtained after summarizing all V4 sequence counts into species groups to overcome the potential problem introduced by over-splitting taxa into reference OTUs. The V4 technique found many taxa that diminished with antibiotic treatment, but few that rebounded (Supplementary Figure S5). Integration of the two technologies, as summarized in Table 1, was enabled by mapping both to the same taxonomy revealing the genera Bacteroides and Parabacteroides to contain PPOs found in both data sets.

The bacterial PPOs that were classified to a recognized genus were then scored from 1 to 5 based on the integrated results across technologies and against public HMP data (Table 1). The purpose was to highlight taxa with the most confirmatory evidence of relevance to human health. Bacteroides and Parabacteroides contained PPOs found using both technologies and were also prevalent in human stools; thus, they scored a 5. Bifidobacterium, Prevotella, Rikenellaceae, Flavobacteriaceae, Blautia, Clostridium, Roseburia, Ruminococcus and Oscillospira all scored 4 since these groups contained PhyloChip PPOs, were all verified as present in the hamster stools by V4, and have been found in human stools. Three families within Sphingobacteriales, namely the Sphingobacteriaceae, Chitinophagaceae and Flexibacteraceae, attained the next highest score of 3 since they have been observed in HMP data but not specifically in HMP stool. It should be mentioned that the HMP 16S rRNA gene surveys were collected with $\sim 5.0 \times 10^{3}$ to $\sim 1.0 \times 10^{5}$ reads per sample, a depth at which presence/absence data is unreliable (Caporaso et al., 2012), suggesting that alternative scoring schemes such as weighting the hybridization data more heavily could be explored in future investigations. A surprising, albeit low-scoring, observation was the occurrence of the archaeal PPOs Euryarchaeota (Halorubrum and MOB7-9) and Crenarchaeota (pSL4).

\section{Discussion}

The goal of this study was to understand the effects of clindamycin treatment on the gut microbiota of golden Syrian hamsters and the impact of microbiome changes on susceptibility of the hamsters to C. difficile colonization, bacterial growth and subsequent disease. We initially observed that fecal extracts, collected from animals 5 days after administration of a single dose of clindamycin, supported 


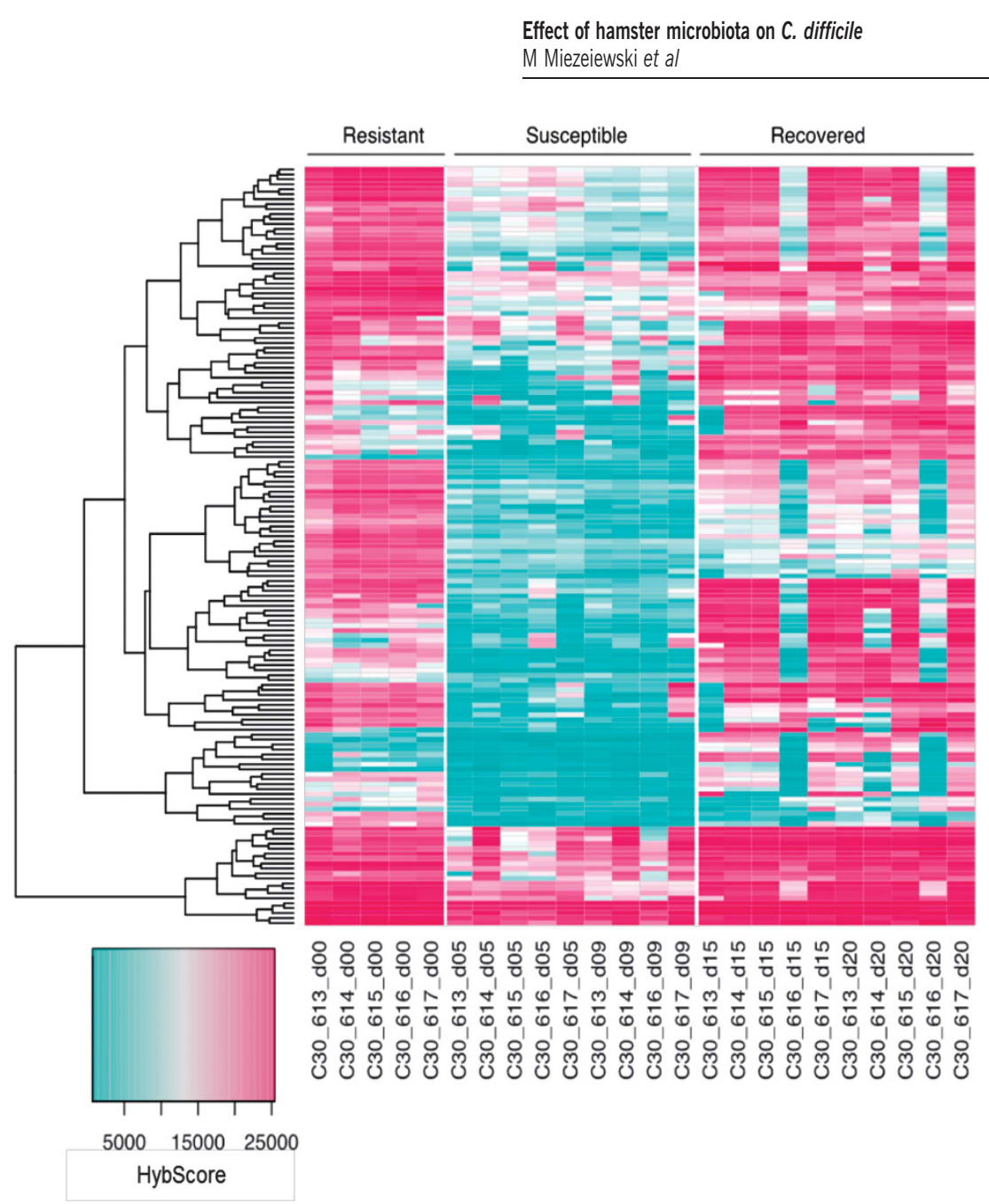

Figure 7 Population dynamics across time points for high dosage clindamycin group for 153 OTUs putatively protective against C. difficile infection. Heat Map coloration represents highest hybridization scores (Hybscores) as red and lowest as blue. Dendrogram at left represents hierarchical clustering of pair-wise Euclidian distances among OTU trajectories.

the germination and growth of $C$. difficile spores on agar plates containing taurocholate to a much greater extent than extracts obtained from animals prior to antibiotic treatment. This observation was manifested by both larger and more numerous clostridial colonies and suggested that residual clindamycin had been cleared from the hamster gut by 5 days after treatment and that the animals were likely susceptible to C. difficile challenge. We next wished to examine this phenomenon in greater detail and to understand both the period of susceptibility of hamsters to challenge as well as the role of various microbial taxa in the rendering hamsters resistant to infection so we developed a novel, broth culture method to investigate the effects of fecal extracts obtained at various time points after antibiotic treatment.

The observation that filtered fecal extracts retained factors inhibitory for the growth of C. difficile was inconsistent with previous reports suggesting that these components were removed by filtration (Borriello and Barclay, 1986). One possible explanation for this discrepancy was the inclusion of taurocholate in the culture medium used for our experiments. While taurocholate has a role in
C. difficile growth through enhancing spore germination, it has also been shown to be metabolized by certain members of the microbiota found in the gut into secondary bile salts (Sorg and Sonenshein, 2008). These secondary bile salts have an inhibitory effect on $C$. difficile growth and germination, as was demonstrated in an ex vivo experiment where the cecal contents collected from mice either pre or post antibiotic treatment were mixed with taurocholate (Wilson, 1983; Giel et al., 2010). The investigators reported that the addition of taurocholate to cecal contents from mice that had not been treated with antibiotics yielded substances that were inhibitory to $C$. difficile growth, while taurocholate-supplemented cecal contents from antibiotic treated mice were permissive for $C$. difficile growth. The authors also determined that the cecal microbiota were metabolizing taurocholate into secondary bile salts that were inhibitory for $C$. difficile growth, and that the presence of the enzymes responsible for converting primary bile salts into secondary bile salts were found in equivalent concentrations in both the cecum and in the feces ( Coleman et al., 1994; Berr et al., 1996; Thomas et al., 2001), an observation 
Table 1 Taxa containing one or more PPO scored from 1 to 5 based on congruence from hybridization and sequencing techniques and prevalence of the taxon in the human microbiome

\begin{tabular}{|c|c|c|c|c|c|c|c|c|c|}
\hline & \multicolumn{2}{|c|}{$\begin{array}{c}\text { Counts of putatively protective } \\
\text { OTUs (PPOs) }\end{array}$} & \multicolumn{2}{|c|}{$\begin{array}{l}\text { Taxon detected in } \\
\text { one or more } \\
\text { samples }\end{array}$} & \multicolumn{2}{|c|}{$\begin{array}{c}\text { HMP prevalence } \\
\text { among stool samples } \\
(\%)\end{array}$} & \multicolumn{2}{|c|}{$\begin{array}{l}\text { HMP prevalence } \\
\text { among all samples } \\
(\%)\end{array}$} & \multirow[b]{2}{*}{ Final score } \\
\hline & Hyb $q<0.05$ & V4 $P<0.05$ & $H y b$ & $V 4$ & $V 1-V 3$ & $V 3-V 5$ & $V 1-V 3$ & $V 3-V 5$ & \\
\hline \multicolumn{10}{|l|}{ Archaea } \\
\hline \multicolumn{10}{|l|}{ Crenarchaeota } \\
\hline pSL4 & 1 & & $\mathrm{Y}$ & & & & & & 1 \\
\hline \multicolumn{10}{|l|}{$\begin{array}{l}\text { puLt } \\
\text { Euryarchaeota }\end{array}$} \\
\hline Halorubrum & 1 & & $\mathrm{Y}$ & & & & & & 1 \\
\hline MOB7-9 & 1 & & $\mathrm{Y}$ & & & & & & 1 \\
\hline \multicolumn{10}{|l|}{ Bacteria } \\
\hline \multicolumn{10}{|l|}{ Actinobacteria } \\
\hline Brevibacterium & 1 & & $\mathrm{Y}$ & & & & 2.4 & 2.9 & 2 \\
\hline Microbacterium & 1 & & $\mathrm{Y}$ & & & & 3.4 & 2.9 & 2 \\
\hline Actinoalloteichus & 1 & & $\mathrm{Y}$ & & & & & & 1 \\
\hline Bifidobacterium & 4 & & $\mathrm{Y}$ & $\mathrm{Y}$ & 1.1 & 3.9 & 1.1 & 1.2 & 4 \\
\hline \multicolumn{10}{|l|}{ Bacteroidetes } \\
\hline Bacteroides & 10 & 2 & $\mathrm{Y}$ & $\mathrm{Y}$ & 100.0 & 100.0 & 42.0 & 56.2 & 5 \\
\hline Parabacteroides & 7 & 6 & $\mathrm{Y}$ & $\mathrm{Y}$ & 89.3 & 89.7 & 17.5 & 24.7 & 5 \\
\hline Prevotella & 5 & & $\mathrm{Y}$ & $\mathrm{Y}$ & 47.6 & 47.0 & 80.0 & 83.1 & 4 \\
\hline Rikenellaceae & 38 & & $\mathrm{Y}$ & $\mathrm{Y}$ & 62.6 & 58.9 & 19.2 & 27.9 & 4 \\
\hline Flavobacteriaceae & 1 & & $\mathrm{Y}$ & $\mathrm{Y}$ & 12.3 & 5.0 & 70.1 & 71.8 & 4 \\
\hline Chitinophagaceae & 1 & & $\mathrm{Y}$ & $\mathrm{Y}$ & & & 2.9 & 1.6 & 3 \\
\hline \multirow[t]{2}{*}{ Flexibacteraceae } & 2 & & $\mathrm{Y}$ & $\mathrm{Y}$ & & & 2.4 & 2.5 & 3 \\
\hline & 1 & & $\mathrm{Y}$ & $\mathrm{Y}$ & & & 2.3 & 2.2 & 3 \\
\hline \multicolumn{10}{|l|}{$\begin{array}{l}\text { Sphingobacteriaceae } \\
\text { CVanobacteria }\end{array}$} \\
\hline Streptophyta & 1 & & $\mathrm{Y}$ & $\mathrm{Y}$ & 0.5 & 0.6 & 8.2 & 18.7 & 4 \\
\hline \multicolumn{10}{|l|}{ Firmicutes } \\
\hline Bacillus & 2 & & $\mathrm{Y}$ & & & 0.3 & 4.9 & 5.6 & 3 \\
\hline Blautia & 1 & & $\mathrm{Y}$ & $\mathrm{Y}$ & 95.2 & 97.8 & 16.1 & 18.7 & 4 \\
\hline Clostridium & 1 & & $\mathrm{Y}$ & $\mathrm{Y}$ & 96.3 & 98.1 & 13.0 & 16.2 & 4 \\
\hline Roseburia & 1 & & $\mathrm{Y}$ & $\mathrm{Y}$ & 97.9 & 95.6 & 18.3 & 19.9 & 4 \\
\hline Ruminococcus & 1 & & $\mathrm{Y}$ & $\mathrm{Y}$ & 93.6 & 78.0 & 14.1 & 17.9 & 4 \\
\hline Bacteroides & 1 & & $\mathrm{Y}$ & $\mathrm{Y}$ & 21.4 & & 1.6 & & 4 \\
\hline Oscillospira & 2 & & $\mathrm{Y}$ & $\mathrm{Y}$ & 96.8 & 97.5 & 14.1 & 17.0 & 4 \\
\hline \multicolumn{10}{|l|}{ Proteobacteria } \\
\hline Rhodospirillaceae & 2 & & $\mathrm{Y}$ & & & & 0.6 & 2.0 & 2 \\
\hline \multicolumn{10}{|l|}{ Spirochaetes } \\
\hline Treponema & 1 & & $\mathrm{Y}$ & & & & & & 1 \\
\hline
\end{tabular}

Abbreviations: HMP, human microbiome project; Hyb, hybridization; OTU, operational taxonomic unit; PPO, putatively protective OTU; Y, yes. Taxa are grouped by kingdom and phylum. In the Archaea, candidate taxa are also presented.

recently supported by meta-metabolomic profiling (Theriot et al., 2014). A recent report by Howerton et al. (2013) described the use of a synthetic derivative of taurocholate, CamSA, which is a competitive inhibitor of taurocholate-induced spore germination that protected mice from challenge with a large number of $C$. difficile spores, further implicating the critical role of bile salts in the regulation of spore germination. While our data suggests a role for microbiome conversion of primary to secondary bile salts in the inhibition of $C$. difficile spore germination and growth, we have not directly measured bile salts in hamster feces and therefore cannot rule out the impact of other soluble factors on $C$. difficile-induced disease.

We subsequently asked if the in vitro evaluation of fecal extracts collected at various time points following antibiotic administration could be used to determine the corresponding in vivo susceptibility of Syrian hamsters to $C$. difficile challenge. Fecal extracts from naïve hamsters were inhibitory for $C$. difficile growth while extracts obtained from clindamycin-treated hamsters supported $C$. difficile growth. Using fecal extracts from samples collected in a longitudinal study, a plot was generated which strongly resembled the theoretical plot of $C$. difficile risk presented by Rupnik et al. (2009) as well as results reported by Abujamel et al. (2013), which demonstrated that stool suspensions collected from patients undergoing antibiotic therapy were permissive to $C$. difficile growth for a period of $\sim 14$ to 21 days after the clearance of the antibiotics.

We next attempted to correlate the growth inhibitory effect of fecal extracts with resistance to challenge with $C$. difficile spores. Because clindamycin-treated hamsters are exquisitely sensitive to challenge with this bacterium, we monitored survival in hamsters immunized with a vaccine 
consisting of toxoids prepared from the two major toxins produced by the organism, $C$. difficile toxin A and toxin B. Animals challenged with the $C$. difficile strain VPI 10463 demonstrated clinical symptoms of disease including a profound decrease in body weight. By monitoring body weight of challenged animals, we were able to determine that the time point at which the hamsters began regaining weight corresponded with the point at which the fecal extracts began to recover their ability to inhibit the growth of C. difficile. This effect was extrapolatable across multiple dose levels of clindamycin. In addition, animals treated with only very low doses of clindamycin ( $1 \mathrm{mg} \mathrm{kg}^{-1}$ ) were uniformly resistant to $C$. difficile challenge, regardless of their immunization status, presumably due to an incomplete disruption of their microbial flora.

We also examined the recovery of the hamsters' resistance to $C$. difficile challenge in naive hamsters to determine the period of microbiome disruption following antibiotic treatment. To do this, we challenged animals either 5, 9, 15 or 20 days following microbiota disruption with clindamycin. We observed a return of the resistance to infection beginning around 15 days post disruption as these animals were less likely to succumb to CDI with lower levels of $C$. difficile colonization detected in surviving animals. Indeed, by 20 days post disruption, $50 \%$ of the animals survived challenge (Figure 5b). The confluence of this result with the in vitro analysis described above suggest that measuring the inhibitory activity of fecal extracts may be a useful surrogate for predicting the in vivo susceptibility of hamsters to CDI.

The clindamycin-induced microbiome perturbation in the hamster model was in general concordance with past reports in mice, where Enterobacteriaceae were observed to rapidly expand and multiple familes within diverse phyla decline (Buffie et al., 2012; Lawley et al., 2012). Our study expands on this knowledge by observing significant changes in vivo over a greater number of phyla and by directly linking the temporal microbiome shifts to $C$. difficile suppression in vitro.

Using the longitudinal molecular profiling data on the hamster microbiome, we found genera of gut bacteria that were suppressed by clindamycin at an early time point and rebounded in abundance by day 15 post clindamycin treatment. The PPOs within Bacteroides, Clostridia and Bifidobacterium were among the groups shown to be negatively affected by clindamycin treatment and these genera are known to produce the enzyme responsible for converting taurocholate into inhibitory secondary bile salts (White et al., 1981; Gustafsson et al., 1998; Ridlon et al., 2006). From our observations, it appears that the protective genera for hamsters intersect but do not co-inside with those for mice, since past reports demonstrate that additional genera outside those listed in Table 1 were able to resolve CDI in C57BL/6 mice (Lawley et al., 2012).
Interestingly, genera associated with susceptibility were also uncovered. For instance, the PhyloChip but not V4 sequencing reported the presence and antibiotic-associated increase in Chloroflexi and Planctomycetes (Supplementary Table S2), known to be rare members of the healthy mammalian microbiome (DeSantis et al., 2006; Yildirim et al., 2010) and which are resistant to antibiotics (Bunge et al., 2008; Cayrou et al., 2010). These findings suggest that a more comprehensive view of population dynamics was achieved by employing the hybridization assay compared with sequencing alone.

The data presented here provide a useful tool for predicting the effect of microbiome disruption and its relation to CDI risk in hamsters. They also suggest that bacteria commonly associated with bile salt metabolism may be responsible for inhibition of $C$. difficile growth in vivo in hamsters. The translation of the hamster model to natural infection in humans could provide some exciting new possibilities in the C. difficile field. By screening patients for susceptibility to CDI, it should be possible to identify a population at greater risk for disease and allow for intervention by microbiome manipulation or immunization with vaccines or monoclonal antibodies to prevent subsequent infection. This technique may also allow the development of a novel screening method for therapeutic agents, which spare beneficial bacteria in the gut hence preserving the resistance to infection in treated individuals.

\section{Conflict of Interest}

All authors are either current or former employees of Merck \& Co or Second Genome Inc and may hold financial interests in one or both companies.

\section{Acknowledgements}

We thank Mark Knower, Robert Trotta, Triana Dalia, Elizabeth Nelson, Linda Carangia, Jennifer Werner and Noelle Dahl for their technical assistance with the C. difficile challenge model.

\section{References}

Abujamel T, Cadnum JL, Jury LA, Sunkesula VC, Kundrapu S, Jump RL et al. (2013). Defining the vulnerable period for re-establishment of Clostridium difficile colonization after treatment of $C$. difficile infection with oral vancomycin or metronidazole. PLoS One 8: e76269.

Berr F, Kullak-Ublick GA, Paumgartner G, Munzing W, Hylemon PB. (1996). 7 alpha dehydroxylating bacteria enhance deoxycholic acid input and cholesterol saturation of bile in patients with gallstones gastroenterology. Gastroenterology 111: 1611-1620.

Borriello SP, Barclay FE. (1986). An in-vitro model of colonisation resistance to Clostridium difficile infection. J Med Micro 21: 299-309.

Buffie CG, Jarchum I, Equinda M, Lipuma L, Gobourne A, Viale A et al. (2012). Profound alterations of intestinal 
microbiota following a single dose of clindamycin results in sustained susceptibility to Clostridium difficile-induced colitis. Infect Immune 80: 62-73.

Bunge M, Wagner A, Fischer M, Andreesen JR, Lechner U. (2008). Enrichment of a dioxin-dehalogenating Dehalococcoides species in two-liquid phase cultures. Environ Microbiol 10: 2670-2683.

Caporaso JG, Lauber C, Walters WA, Berg-Lyons D, Lozupone CA, Turnbaugh PJ et al. (2011). Global patterns of $16 \mathrm{~S}$ rRNA diversity at a depth of millions of sequences per sample. Proc Natl Acad Sci USA 15: 4516-4522.

Caporaso JG, Paszkiewicz K, Field D, Knight R, Gilbert JA. (2012). The Western English Channel contains a persistent microbial seed bank. ISME J 10: 1089-1093.

Cayrou C, Raoult D, Drancourt M. (2010). Broad-spectrum antibiotic resistance of Planctomycetes organisms determined by Etest. J Antimicrob Chemother 65: 2119-2122.

Coleman JP, Hudson LL, Adams MJ. (1994). Characterization and regulation of the NADP-linked 7 alphahydroxysteroid dehydrogenase gene from Clostridium sordellii. J Bacteriol 176: 4865-4874.

DeSantis TZ, Hugenholtz P, Larsen N, Rojas M, Brodie EL, Keller K et al. (2006). Greengenes, a chimera-checked $16 \mathrm{~S}$ rRNA gene database and workbench compatible with ARB. Appl Environ Microbiol 72: 5069-5072.

Friedman J, Alm EJ. (2012). Inferring correlation networks from genomic survey data. PLoS Comput Biol 8: e1002687.

Giel JL, Sorg JA, Sonenshein AL, Zhu J. (2010). Metabolism of bile salts in mice influences spore germination in Clostridium difficile. PLoS One 5: 1-7.

Gustafsson A, Norin E, Midtvedt T. (1998). Effect of clindamycin given alone or with Lactobacillus delbrueckii and Streptococcus thermophilus on 7 alpha dehydroxylation of bile acids in rats. Clin Microbiol Infect 4: 594-598.

Hazen TC, Dubinsky EA, DeSantis TZ, Andersen GL, Piceno YM, Singh N et al. (2010). Deep-sea oil plume enriches indigenous oil-degrading bacteria. Science 330: 204-208.

Howerton A, Patra M, Abel-Santos E. (2013). A new strategy for the prevention of Clostridium difficile infection. I Infect Dis 207: 1498-1504.

Human Microbiome Project Consortium (2012). Structure, function and diversity of the healthy human microbiome. Nature 486: 207-214.

Kuczynski J, Stombaugh J, Walters WA, González A, Caporaso JG, Knight R. (2011). Using QIIME to analyze 16S rRNA gene sequences from microbial communities. Curr Protoc Microbiol ; Chapter 10:Unit 10.7. doi: 10.1002/0471250953.bi1007s36.

Lawley TD, Clare S, Walker AW, Stares MD, Connor TR, Raisen C et al. (2012). Targeted restoration of the intestinal microbiota with a simple, defined bacteriotherapy resolves relapsing Clostridium difficile disease in mice. PLoS Pathogens 8: e1002995.

Long Sterling K, Williams OB. (1958). Method for removal of vegetative cells from bacterial spore preparations. J Bacteriol 76: 332-332.

Lozupone C, Hamady M, Knight R. (2006). UniFrac-an online tool for comparing microbial community diversity in a phylogenetic context. BMC Bioinformatics 7: 371.
McDonald D, Price MN, Goodrich J, Nawrocki EP, DeSantis TZ, Probst A et al. (2012). An improved Greengenes taxonomy with explicit ranks for ecological and evolutionary analyses of bacteria and archaea. ISME J 6: 610-618.

Penders J, Vink C, Driessen C, London N, Thijs C, Stobberingh EE. (2005). Quantification of Bifidobacterium spp., Escherichia coli and Clostridium difficile in faecel samples of breast-fed and formula-fed infants by real-time PCR. FEMS Microbiol Lett 243: 141-147.

Poutanen SM, Simor AE. (2004). Clostridium difficile-associated diarrhea in adults. Can Med Assoc J 171: 51-58.

Edgar RC. (2010). Search and clustering orders of magnitude faster than BLAST. Bioinformatics 26: 2460-2461.

Ridlon JM, Kang DJ, Hylemon PB. (2006). Bile salt biotransformations by human intestinal bacteria. J Lipid Res 47: 241-259.

Rupnik M, Wilcox MH, Gerding DN. (2009). Clostridium difficile infection: new developments in epidemiology and pathogenesis. Nat Rev Microbiol 7: 526-536.

Sambol SP, Tang JK, Merrigan MM, Johnson S, Gerding DN. (2001). Infection of hamsters with epidemiologically important strains of Clostridium difficile. Infect Dis 183: 1760-1766.

Schloss PD, Westcott SL, Ryabin T, Hall JR, Hartmann M, Hollister EB et al. (2009). Introducing mothur: opensource, platform-independent, community-supported software for describing and comparing microbial communities. Appl Environ Microbiol 75: 7537-7541.

Sorg JA, Sonenshein AL. (2008). Bile salts and glycine as co-germinants for Clostridium difficile spores. J Bacteriol 190: 2505-2512.

Tamaki H, Tanaka Y, Matsuzawa H, Muramatsu M, Meng XY, Hanada $S$ et al. (2011). Armatimonas rosea gen. nov., sp. nov., of a novel bacterial phylum, Armatimonadetes phyl. nov., formally called the candidate phylum OP10. Int J Syst Evol Microbiol 61: 1442-1447.

Theriot CM, Koenigsknecht MJ, Carlson Jr PE, Hatton GE, Nelson AM, Li B et al. (2014). Antibiotic-induced shifts in the mouse gut microbiome and metabolome increase susceptibility to Clostridium difficile infection. Nat Commun 5: 3314.

Thomas LA, Veysey MJ, French G, Hylemon PB, Murphy GM, Dowling RH. (2001). Bile acid metabolism by fresh human colonic contents: a comparison of cecal versus faecal samples. Gut 49: 835-842.

van Nood E, Vrieze A, Nieuwdorp M, Fuentes S, Zoetendal EG, de Vos WM et al. (2013). Duodenal infusion of donor feces for recurrent Clostridium difficile. NEJM 368: 407-415

White BA, Cacciapuoti AF, Fricke RJ, Whitehead TR, Mosbach EH, Hylemon PB. (1981). Cofactor requirements for 7 alpha dehydroxylation of cholic and chenodeoxycholic acid in cell extracts of the intestial anaerobic bacterium, Eubacterium species V.P.I. 12708. J Lipid Res 22: 891-898.

Wilson KH. (1983). Efficiency of various bile salt preparations for stimulation of Clostridium difficile spore germination. J Clin Microbiol 18: 1017-1019.

Yildirim S, Yeoman CJ, Sipos M, Torralba M, Wilson BA, Goldberg TL et al. (2010). Characterization of the fecal microbiome from non-human wild primates reveals species specific microbial communities. PLoS One 5: e13963. 